\title{
CHARA Michigan phase-tracker (CHAMP): design and fabrication
}

David H. Berger, John D. Monnier, Rafael Millan-Gabet, Theo A. ten Brummelaar, Philip Muirhead, et al.

David H. Berger, John D. Monnier, Rafael Millan-Gabet, Theo A. ten Brummelaar, Philip Muirhead, Ettore Pedretti, Nathalie Thureau, "CHARA Michigan phase-tracker (CHAMP): design and fabrication," Proc. SPIE 6268, Advances in Stellar Interferometry, 62683K (28 June 2006); doi: 10.1117/12.672168

Event: SPIE Astronomical Telescopes + Instrumentation, 2006, Orlando, Florida , United States 


\title{
CHARA Michigan Phase-tracker (CHAMP): Design and Fabrication
}

\author{
David H. Berger ${ }^{a}$, John D. Monnier ${ }^{a}$, Rafael Millan-Gabet ${ }^{b}$, Theo A. ten Brummelaar $^{c}$, Philip \\ Muirhead $^{d}$, Ettore Pedretti ${ }^{a}$, Nathalie Thureau ${ }^{a}$ \\ ${ }^{a}$ University of Michigan, Department of Astronomy, 500 Church St, Ann Arbor, MI 48109, \\ USA; \\ ${ }^{b}$ Michelson Science Center, California Institute of Technology, 770 South Wilson Avenue, \\ Pasadena, CA 91125, USA; \\ ${ }^{c}$ The CHARA Array, Mount Wilson Observatory, Mount Wilson, CA 91023, USA; \\ ${ }^{d}$ Cornell University, 110 Space Sciences Building, Ithaca, NY 14853, USA
}

\begin{abstract}
We present the design for a near-infrared (JHK) fringe tracker to be used at the CHARA Array, a long baseline optical interferometer located at Mount Wilson Observatory. The CHARA Michigan Phase-tracker (CHAMP) is being fabricated and tested at the University of Michigan and will be transported to the CHARA Array for general use. CHAMP is separate from the science combiners and can therefore be optimized for fringe tracking. It will modulate around fringe center by $1-2 \lambda$ at up to $500 \mathrm{~Hz}$ and calculate phase offsets in real-time using a modified 'ABCD' method. Six pair-wise Mach-Zehnder combiners will phase the entire Array. We give an overview of the optical layout and discuss our design strategy. Components such as the path-length modulators, low-OH fiber transport system, 1024x1024 HAWAII-1 detector, and control computer are discussed.
\end{abstract}

Keywords: fringe tracking, long baseline optical interferometry

\section{INTRODUCTION}

The CHARA Michigan Phase-tracker (CHAMP) is a near-infrared fringe tracker (FT) being designed and fabricated by our team at the University of Michigan for use at Georgia State University's (GSU) Center for High Angular Resolution Astronomy (CHARA) Array. The CHARA Array is a "Y"-shaped long baseline interferometer at Mount Wilson Observatory operating in the visible and near-infrared. ${ }^{1}$ There is currently no FT for coherent integration at the CHARA Array, but passive fringe packet tracking through software exists for each of the science combiners. CHAMP will provide longer coherent integration times for the science combiners, particularly the newly commissioned Michigan InfraRed Combiner (MIRC) (Monnier et al., these proceedings).

The primary goal of CHAMP is to provide real-time phase tracking error estimates. We subscribe to the idea that an FT should be separate from the science instruments, an idea that was part of the original design plan for the CHARA Array. ${ }^{2}$ Under this construct, the FT and science combiners can be uniquely optimized and independently developed. The task of measuring well calibrated, high precision visibilities and/or phase closures is therefore left to the science instrument, and the FT can be designed primarily for increased sensitivity. The Very Large Telescope Interferometer (VLTI) has also adopted a similar philosophy for their multi-beam combiner AMBER. ${ }^{3}$

Further author information: (Send correspondence to D.H.B.)

D.H.B.: E-mail: dhberger@umich.edu, Telephone: 17347644160

J.D.M.: E-mail: monnier@umich.edu

R.M-G.: E-mail: rafael@ipac.caltech.edu

T.A.tB: E-mail: theo@chara-array.org

P.M.: E-mail: muirhead@astro.cornell.edu

E.P.: E-Mail: epedrett@umich.edu

N.T.: E-Mail: thureau@mrao.cam.ac.uk

Advances in Stellar Interferometry, edited by John D. Monnier,

Markus Schöller, William C. Danchi, Proc. of SPIE Vol. 6268,

62683K, (2006) - 0277-786X/06/\$15 - doi: 10.1117/12.672168

Proc. of SPIE Vol. 6268 62683K-1 
CHAMP will use a modified 'ABCD' method of phase tracking, similar to that used by the Mark III interferometer, ${ }^{4}$ the Navy Prototype Optical Interferometer (NPOI), ${ }^{5}$ and the Keck Interferometer (KI) ${ }^{6}$ By path-modulating the fringe by at least one wavelength at a rate faster than the atmospheric coherence time (approximately $10 \mathrm{~ms}$ ), one can servo out the fringe phase fluctuations using the delay lines. The effect is to lock the fringe center onto the science detector to within a fraction of a wavelength allowing for coherent integration.

In this paper, we will describe the scientific gains CHAMP will provide, particularly with MIRC and the visible beam combiner $(\S 2)$. Section 3 is a survey optics and optical hardware that make up the input optics, beam combiner, fiber optics, and detector. This section will also describe the computer system and outline the control flow between subsystems. Section 4 outlines the current schedule for design, testing, and integration with the CHARA Array.

\section{MOTIVATION}

CHAMP was designed primarily to support the scientific goals of MIRC, ${ }^{7}$ an image-plane, spectroscopic combiner. The advantages of this all-in-one combiner is that it yields 15 visibilities and 20 closure phases from the 6 CHARA telescopes simultaneously. The disadvantage over classical two-beam combiners is the reduced sensitivity and increased readout time from spreading the light over many pixels $(\sim 50 \times 120)$. Although the true magnitude limit of MIRC is yet to be determined, commissioning observations demonstrated the ability to track on an $\mathrm{H}=3.0$ star ( $\nu$ And) under poor seeing conditions (Monnier et al., these proceedings). With the addition of CHAMP we expect a several magnitude improvement in the J-, $\mathrm{H}-$, and $\mathrm{K}_{s}$-bands.

As outlined in Ref. 7, MIRC/CHARA's high resolution interferometric imaging enables some unique science capabilities, specifically probing young stellar objects and the direct detection of faint companions. Sub-AU imaging of the inner accretion disks around young stellar objects is an important yet poorly understood steppingstone to understanding planet formation. Also, high precision closure phase measurements are uniquely suited to detect faint companions such as "hot Jupiters." These low visibility or high dynamic range sources require the improved SNR capability of CHAMP.

A distinct advantage of the CHARA Array's 1-meter telescopes and long baselines is the ability to measure stellar diameters of small, intrinsically faint stars such as M-dwarfs. Recently, the diameters of six M-dwarf have been measured by the two-beam, pupil-plane, near-infrared combiner (known as CHARA "Classic" ${ }^{8}$ ) at the CHARA Array in the $\mathrm{K}_{s}$-band. ${ }^{9}$ Five of the diameter measurements are new and represent over $40 \%$ of the few M-dwarfs measured by interferometry. Future plans call for an extension to visible wavelengths where not only is the resolution higher but stronger limb-darkening will reveal new information about the stellar atmospheres of cool stars. However, M-dwarfs are intrinsically dimmer in the visible than in the near-infrared, and the use of CHAMP is required for increased sensitivity and higher SNR visibility measurements.

\section{OPTICS AND OPTO-MECHANICS}

Design and fabrication of CHAMP has been ongoing for the past year at the University of Michigan. Upon completion and testing, CHAMP will be transported to the CHARA Array and integrated into the existing infrastructure. The future location of CHAMP within the Beam Combination Laboratory (BCL) is shown in Fig. 1. Before entering the BCL, the collimated beams from each telescope have been compressed to $\varnothing 20 \mathrm{~mm}$ and optionally corrected for longitudinal dispersion. ${ }^{10}$ Each beam is subsequently split into visible and near-infrared components $\left(\lambda_{\text {split }}=1 \mu \mathrm{m}\right)$. The resulting twelve beams enter the BCL at the Northeast corner and have a 11.2 inch phase delay between adjacent beams. The visible beams pass eastward of the optical table and are untouched by the CHAMP optics.

This section is a tour of the optics and their associated hardware. A schematic layout of CHAMP is shown in Fig. 2. 


\section{FLUOR}

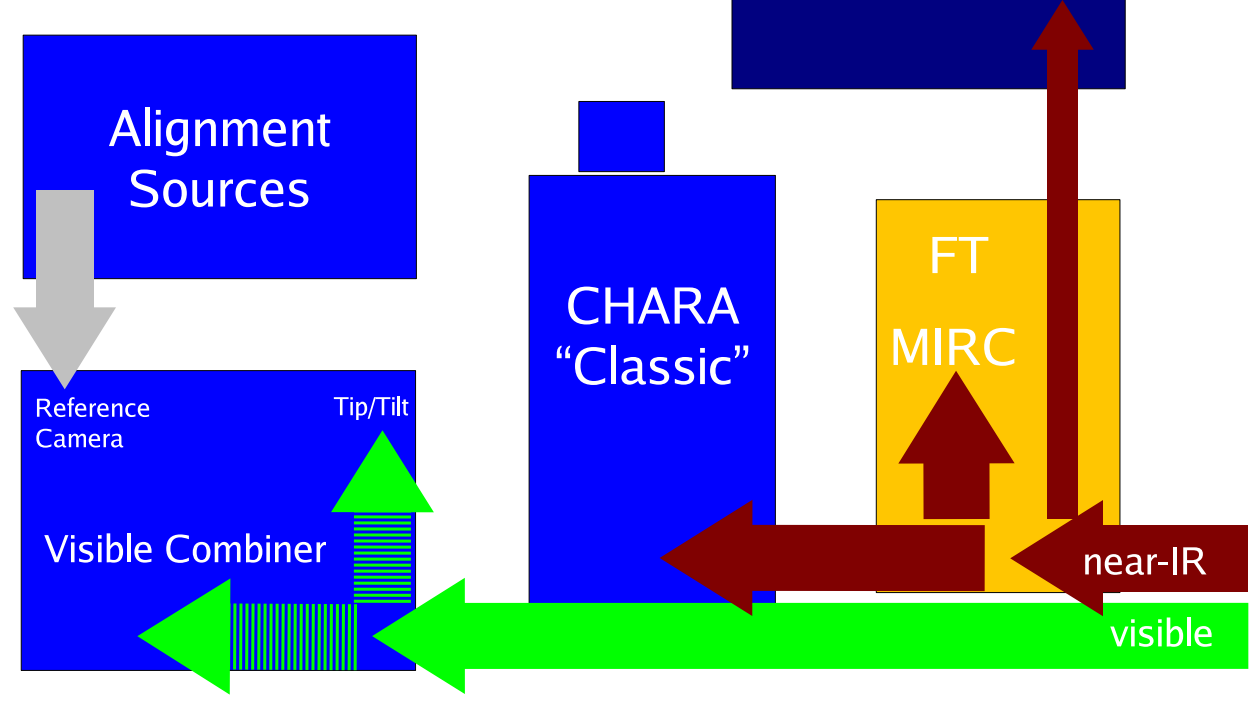

Figure 1. Block diagram of the CHARA Array Beam Combination Laboratory. North is to the right and East is down. Each set of six beams (visible and near-infrared) are represented by the large arrows. The narrow arrow represents the two beams use by FLUOR.

\subsection{Input Optics}

The input optics are first to encounter the near-infrared beams entering the BCL. This is where CHAMP is phased both internally and to the science instrument. Although CHAMP has been designed to match the zero OPD of the other beam combiners, additional adjustment will most likely be required. The input optics will be on linear stages (25.4 mm travel) with encoded actuators. The small AOI (see below) minimizes misalignment when moving the stages.

Beam splitters divide light between the science instrument and CHAMP. The reflected portion is angled down $\left(3^{\circ} \mathrm{AOI}\right)$ to the 4.5-inch beam height of CHAMP and the transmitted part continues to the science instrument. The lower beam height keeps CHAMP below the FLUOR pickoff beams, and the small AOI reduces the deviation of the transmitted beam. There will be three sets of beam splitters depending of science combiner's needs. The first is a 50/50 gray split over $\mathrm{J}-, \mathrm{H}-$, and $\mathrm{K}_{s}$-bands. The second and third beam splitter sets are dichroics: one set that reflects in the $\mathrm{J}$ - and $\mathrm{H}$-bands while transmitting in the $\mathrm{K}_{s}$-band and the other that reflects in the $\mathrm{K}_{s}$-band while transmitting in the $\mathrm{J}$ - and $\mathrm{H}$-bands.

Each beam splitter will be housed in an aluminum shell that can easily be placed in the optics mounts. This facilitates switching between splitter sets and minimizing direct contact with the substrate and coating. The substrates have been specified to be $\varnothing 25.4 \times 4 \mathrm{~mm} \mathrm{CaF}_{2}$ disks with a few arcminute wedge to remove back-reflected "ghost" images out of the beam. All optics have an anti-reflective coating on one side.

\subsection{Path-Length Modulation}

CHAMP combines the beams in a cyclical, pair-wise fashion $(1+2,2+3 \ldots 5+6,6+1)$ so path-length modulation is only needed for every other beam to uniquely identify delay line offsets and direction. Dithering is accomplished by modulating the collimated beam via a mirror mounted on a piezoelectric actuator. We used the PZ 8 D12 piezo with an $8 \mu$ m maximum amplitude driven by the ENV 150 amplifier.* The piezo will be driven

\footnotetext{
*Piezosystem Jena, Inc.
} 


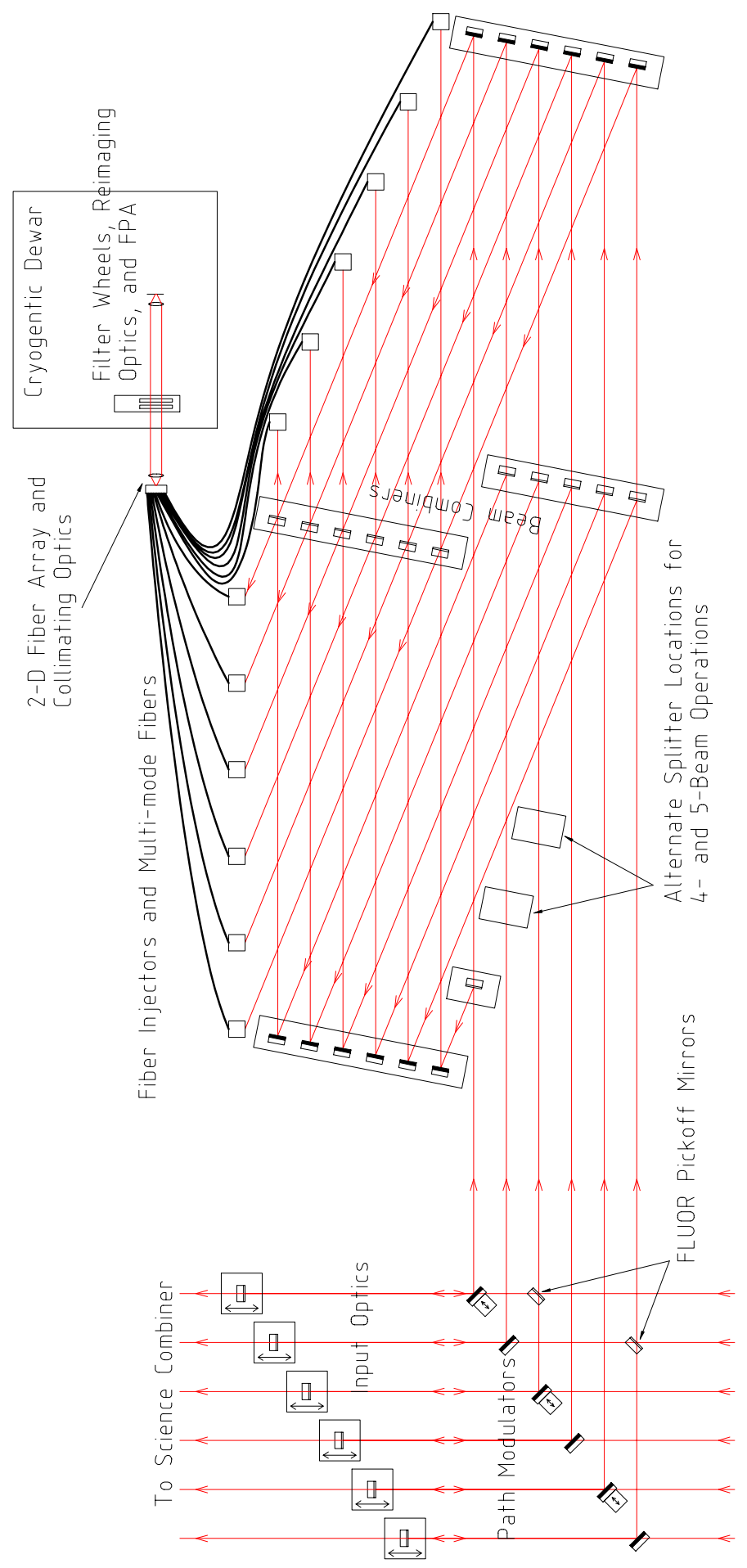

Figure 2. CHAMP schematic. This schematic is rotated $90^{\circ}$ clockwise with respect to Fig. 1 such that North is down and East is to the left. Hardware positions are shown to scale but, for clarity, their representations are not. Note that the input beam from the telescopes passes above the path-modulators and is reflected downward ( $3^{\circ}$ AOI) by the input optics. 


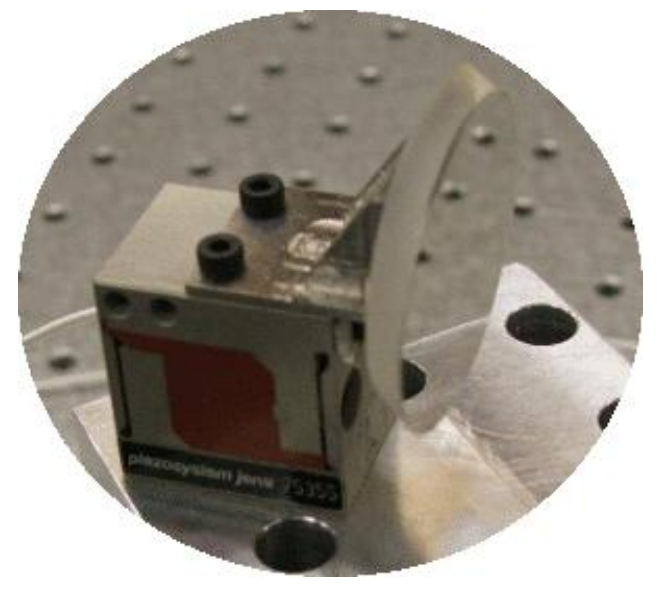

Figure 3. The piezo and mirror prototype. The piezo is a PZ 8 D12 from Piezosystem Jena, Inc. with a custom invar angle bracket and a $22.4 \times 31.5 \times 3.5 \mathrm{~mm}, \lambda / 10$ elliptical mirror.

at 100 to $500 \mathrm{~Hz}$, depending on the source brightness and seeing conditions. Accounting for the 45 degree angle of incidence of the beam with the mirror, the total dither range will be up to 1.6 microns, or $2 \lambda$ at $\mathrm{K}_{s}$-band. Discussion of the modulation voltage source is in $\S 3.5$.

A custom-designed angle-bracket adapts a $22.4 \times 31.5 \times 3.5$ - $\mathrm{mm}, \lambda / 10$ elliptical mirror ${ }^{\dagger}$ to the top of the piezo actuator (Fig. 3). The optical axis of the mirror is roughly aligned with the modulation direction. The bracket is made of invar, a iron-nickel alloy with a low coefficient of thermal expansion at room temperature. We chose this material to minimize stresses on the mirror by the bracket due to temperature changes in the laboratory and during adhesive curing. The mirror is adhered to the bracket using Hysol EA 9313, a two component paste adhesive used specifically because of its flexible bond, again to minimize stress on the mirror.

The piezo is mounted via a custom-made adapter plate onto stacked goniometers that have a common rotation axis height $\ddagger$. The rotation axes of the goniometers are aligned with the incoming and reflected beams in order to simplify alignment. The goniometers are mounted onto a 2"-diameter aluminum post, which is held to the table with a custom-made collar allowing for rough rotation alignment.

We used a classical two-arm Michelson interferometer to check the performance of our path-length modulator designs. A signal generator sent a sine-modulated voltage to the piezo amplifier, and fringes from a HeNe laser $(\lambda=632.8 \mathrm{~nm})$ were detected using a photodiode. Both the amplifier output and the photodiode voltages were simultaneously monitored using an oscilloscope. Sample output is shown in Fig. 4. By incrementally adjusting the modulation frequency, we were able to visually note when were modulating at the resonant frequency by seeing an abrupt increase in the number of HeNe fringes. The final design shown in Fig. 3 had a resonant frequency of approximately $1.5 \mathrm{kHz}$, well above the planned operating frequency range (100-500 Hz). The angle bracket is designed to be light ( $50 \mathrm{~g}$ including mirror, adhesive, and hardware) and has rear supports to suppress additional vibrational modes. The biggest contributors to maintaining a high resonant frequency were the large, 2-inch diameter post and adapter plate.

To maximize sensitivity, the final modulation pattern with be a saw-tooth with rounded edges and is likely to excite mechanical resonances at lower frequencies. Further work will include calibrating phase measurements for different modulation patterns, frequencies, strokes.

\subsection{Beam Combination}

CHAMP employs a series of six Mach-Zehnder pupil-plane beam combiners, each nominally phased with the visible beam combiner, the zero path difference reference for all instruments at CHARA. To achieve this, the

\footnotetext{
${ }^{\dagger}$ Linos Photonics, Inc.

$\ddagger$ Thorlabs
} 


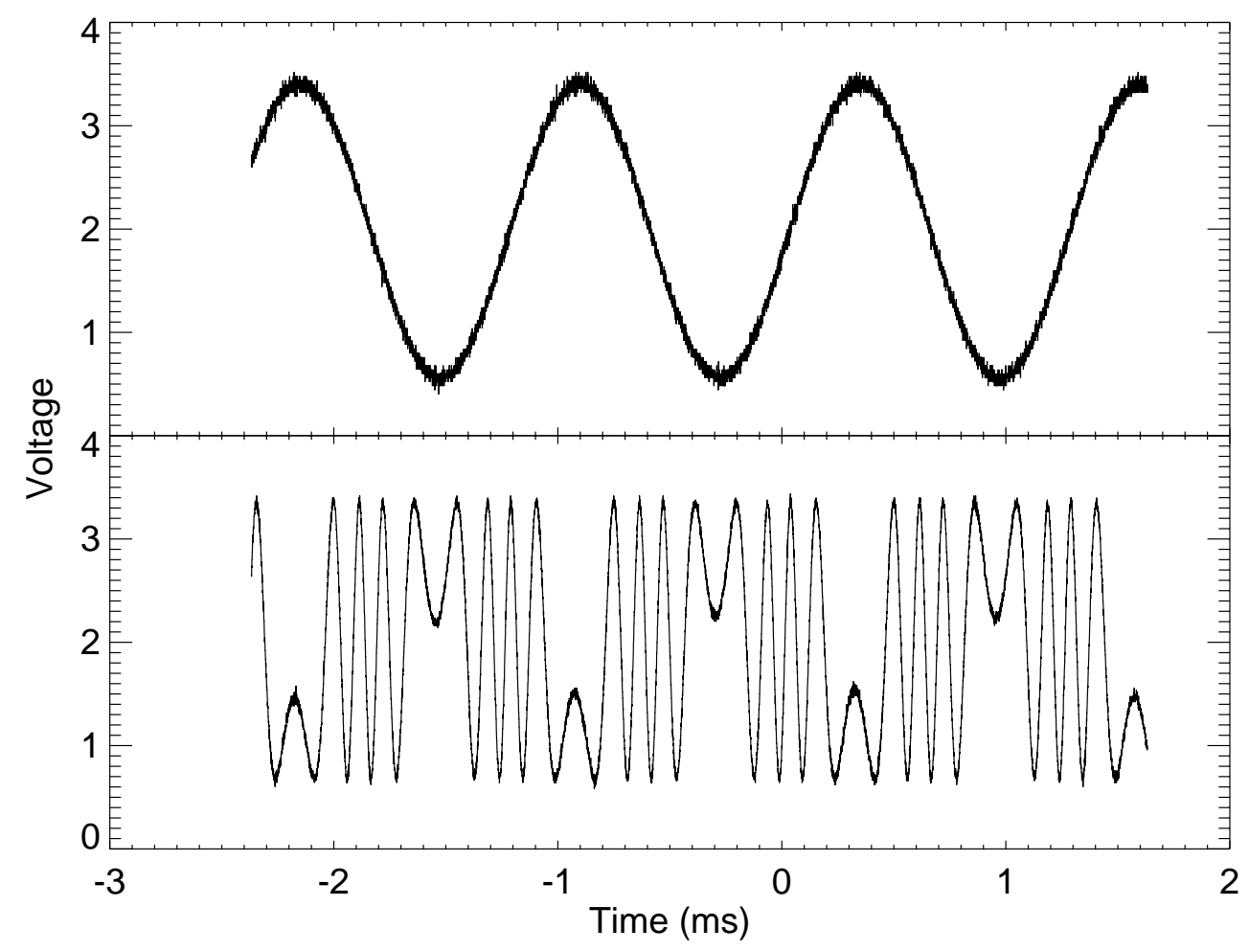

Figure 4. Top: Amplifier output from an $800-\mathrm{Hz}$ voltage modulation signal. Bottom: HeNe fringes as detected by the photodiode.

AOI was calculated from its relation to the ratio of the phase delay $(d)$ to beam spacing $(b)$ :

$$
\frac{d}{b}=\cot (2 \mathrm{AOI})-\csc (2 \mathrm{AOI})
$$

Equation (1) assumes beams are incident on all surfaces at the same angle and yields the most compact design. For $b=2$ inches and $d=10.2$ inches ( 1 inch is removed by changing the beam spacing from 3 to 2 inches), the $\mathrm{AOI}$ is $11.09^{\circ}$.

The beam splitters are $\varnothing 25.4 \times 4 \mathrm{~mm}$ thick low-OH fused silica disks with a 50/50 gray coating. A 30 arcminute wedge removes ghost reflections. Each optic is individually steerable but shares a monolithic base for rough alignment(indicated by large rectangles around the optics in Fig. 2). The exception to this is the beam 6 beam splitter, which can be moved to alternate locations to accommodate using fewer than six beams while maintaining the wrap-around combination with beam 1 .

\subsection{Fiber Transport and Detection}

After the beams are combined, the twelve outputs from both sides of the beam combiners are injected into multi-mode fibers, each of which have a $50-\mu \mathrm{m}$ core and a numerical aperture of 0.12 (equivalent to f/4.2). The use of multi-mode fibers allows the beams to be separately reconfigured for imaging on to the focal plane array (FPA) detector. Fiber lengths will be kept to 1 meter to minimize focal length degradation ${ }^{11}$ and attenuation.

The output ends of the fibers will be held in a custom 2-D array in a pattern optimized for fast readout of the FPA, a $1 \mathrm{k} \times 1 \mathrm{k}$ Hawaii- $1 \mathrm{HgCdTe}$ detector. $\S$ The FPA has four readout amplifiers with a $90^{\circ}$ rotation in

\footnotetext{
${ }^{\S}$ Rockwell Scientific
} 


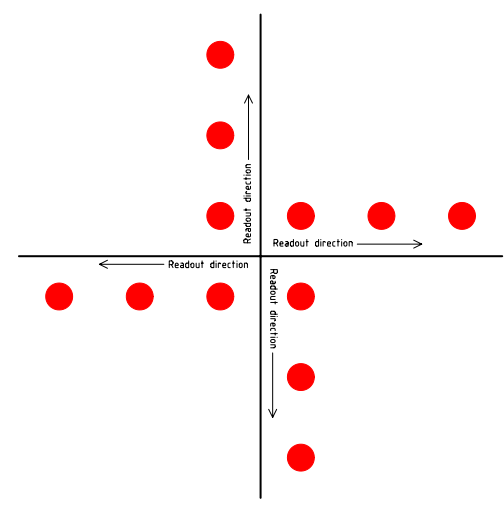

Figure 5. Image pattern on the FPA with four readout amplifier. Spot spacing is shown to be roughly 3 times the diffraction limit spot size.

readout direction between quadrants. Therefore, if the spots are imaged onto the FPA in the pattern shown in Fig. 5, only one line will have to be read.

The detector will be housed, along with two filter wheels, in a cryogenic dewar $\%$. The filter wheels are designed or each to have slots for four 2-inch square filters. The filter wheels will be controlled via serial port, and serial communications are handled via the NPort 5610-8 || 8-port serial-over-IP controller, which is shared with the MIRC instrument.

\subsection{Computer and Signal Control}

The primary tasks of the CHAMP computer are to synchronize the path modulation with the readout of the nearinfrared detector, calculate the fringe phase offsets, and send the offsets to the delay lines. This has to be done deterministically (i.e. in "hard real-time"), and therefore successful completion of the scheduled tasks requires low latency. The MIRC computer has already met these requirements (Pedretti et al., these proceedings), and we have followed a similar development path for the CHAMP computer. In fact, the CHAMP computer is a clone of the one used with MIRC, except the dual Xeon processors are each $3.0 \mathrm{GHz}$ instead of $2.8 \mathrm{GHz}$. The MIRC computer operates under Linux with the RTAI Fusion real-time extension. The CHAMP computer operating system is similar, however the development of RTAI Fusion has since branched off and renamed to Xenomai ${ }^{* *}$.

The CHAMP computer is central to FT servo loop, and Fig. 6 illustrates how it mediates communications between subsystems. The near-infrared beams are phased by the fast delay lines and then split between the science instrument and CHAMP. The science instrument can optionally send a group delay offset at low bandwidth (approximately $1 \mathrm{~Hz}$ ) to CHAMP. As discussed in the previous sections, the beams sent to CHAMP are path modulated, interfered pair-wise, injected into fibers, and arranged onto the detector in a pattern set by the 2-D fiber array. The detector turns photons into electrons ( $\mathrm{QE} \sim 60 \%$ ). The signal is then amplified, converted from analog to digital, and sent to a custom PCI interface card ${ }^{\dagger \dagger}$ inside the CHAMP computer. The fringe visibility and phase are computed, and the offset is sent to the VME computer, which controls the position and velocity of the fast delay lines. The offset position of the fast delay line shifts the fringe position on the science and CHAMP detectors and the loop is completed.

A digital-to-analog converter (DAC) PCI card (PCI-6711) connected to a multi-port breakout box (BNC$2110)$ generates the analog signal to the amplifiers driving the piezo actuators. The modulation signal is pre-programmed onto the DAC and thus does not tap any resources from the computer during operation. In sync with the modulation signal, the breakout box will also trigger the camera readout.

\footnotetext{
"designed and fabricated by IR Labs

"Moxa

${ }^{* *}$ http://www.xenomai.org

${ }^{\dagger \dagger}$ Astronomical Research Cameras (Robert Leach)

${ }^{\ddagger \ddagger}$ National Instruments
} 


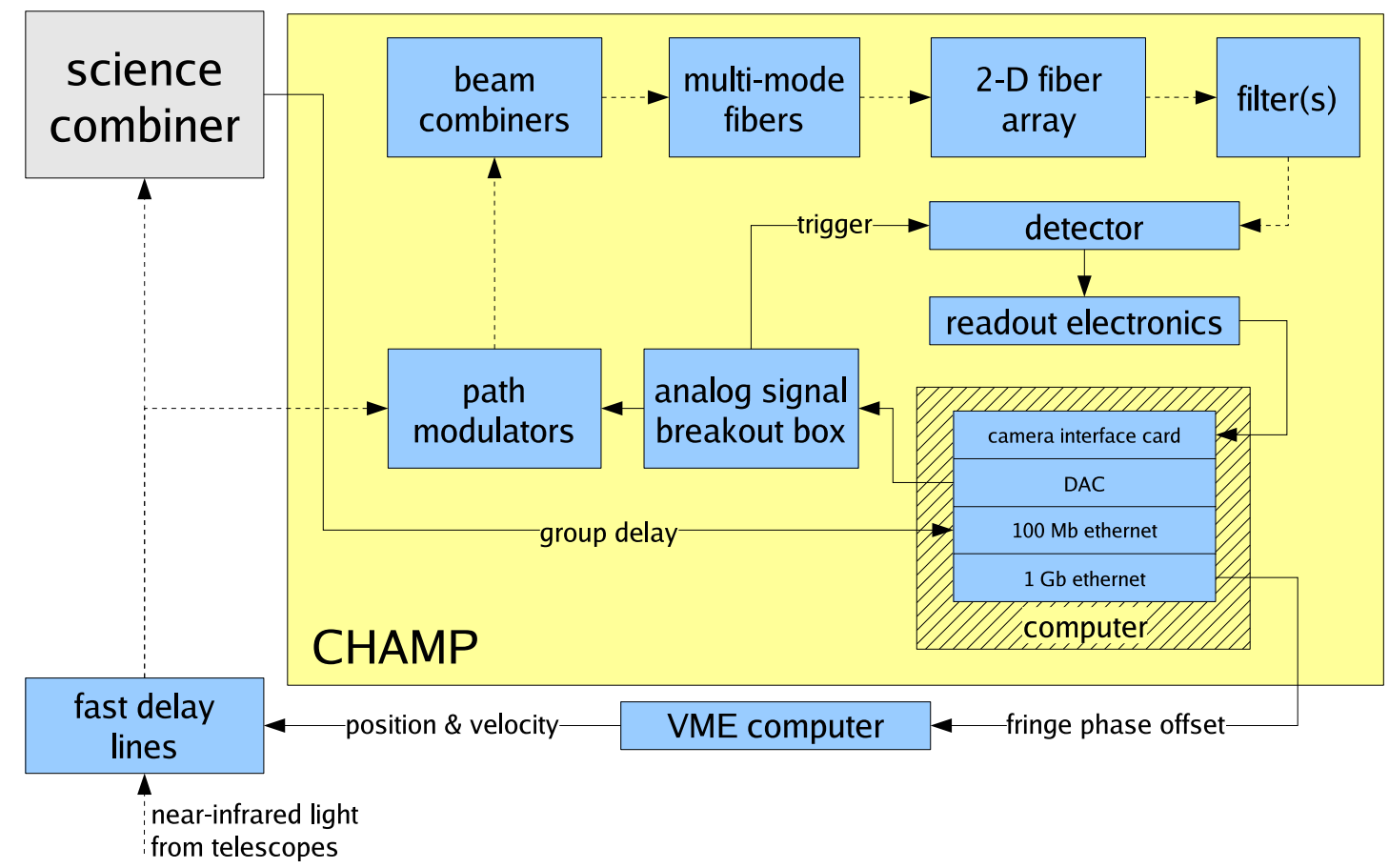

Figure 6. Signal Control Diagram. Dotted lines represent photons and solid lines represent electrons.

\section{SCHEDULE}

We are currently in the design and fabrication phase of CHAMP. By the end of Spring 2006 we expect to finish the optical and mechanical fabrication. This means all glass substrates, coatings, and off-the-shelf opto-mechanical mounts will be ordered. Custom design mounts, such as those for the elliptical path-modulator optics, will have been designed, fabricated, and tested. In the Summer 2006, we expect to test the camera electronics and crygenic dewar. At that time, we will setup the CHAMP optics in the laboratory in Michigan. During the Fall and Winter of 2006, we will integrate the camera with the combiner and close the loop with artificial turbulence. We will also begin upgrades to the CHARA VME computers to accept offsets to fast delay line. The CHARA delay line hardware and software are similar to those used by PTI and KI, and software hooks already exist to accommodate active fringe tracking. Finally, in Summer/Fall 2006, CHAMP will be transported to the CHARA Array for integration and sky testing.

\section{ACKNOWLEDGMENTS}

The fringe tracker funding, including financial support for DHB, was provided by the National Science Foundation through grant AST-0352723.

\section{REFERENCES}

1. T. A. ten Brummelaar, H. A. McAlister, S. T. Ridgway, W. G. Bagnuolo, N. H. Turner, L. Sturmann, J. Sturmann, D. H. Berger, C. E. Ogden, R. Cadman, W. I. Hartkopf, C. H. Hopper, and M. A. Shure, "First Results from the CHARA Array. II. A Description of the Instrument," ApJ 628, pp. 453-465, July 2005.

2. T. A. ten Brummelaar, "Visible Light Fringe Tracking," tech. rep., Georgia State University/CHARA, 1993. http://www.chara.gsu.edu/CHARA/Reports/appendixi.pdf. 
3. M. Gai, S. Menardi, S. Cesare, B. Bauvir, D. Bonino, L. Corcione, M. Dimmler, G. Massone, F. Reynaud, and A. Wallander, "The VLTI fringe sensors: FINITO and PRIMA FSU," in New Frontiers in Stellar Interferometry, Proceedings of SPIE Volume 5491. Edited by Wesley A. Traub. Bellingham, WA: The International Society for Optical Engineering, 2004., p.528, W. A. Traub, ed., pp. 528-+, Oct. 2004.

4. M. Shao, M. M. Colavita, B. E. Hines, D. H. Staelin, and D. J. Hutter, "The Mark III stellar interferometer," A\&A 193, pp. 357-371, Mar. 1988.

5. J. A. Benson, D. Mozurkewich, and S. M. Jefferies, "Active optical fringe tracking at the NPOI," in Proc. SPIE Vol. 3350, p. 493-496, Astronomical Interferometry, Robert D. Reasenberg; Ed., R. D. Reasenberg, ed., pp. 493-496, July 1998.

6. G. Vasisht and M. M. Colavita, "Differential phase interferometry with the Keck telescopes," in New Frontiers in Stellar Interferometry, Proceedings of SPIE Volume 5491. Edited by Wesley A. Traub. Bellingham, WA: The International Society for Optical Engineering, 2004., p.567, W. A. Traub, ed., pp. 567-+, Oct. 2004.

7. J. D. Monnier, J.-P. Berger, R. Millan-Gabet, and T. A. Ten Brummelaar, "The Michigan Infrared Combiner (MIRC): IR imaging with the CHARA Array," in New Frontiers in Stellar Interferometry, Proceedings of SPIE Volume 5491. Edited by Wesley A. Traub. Bellingham, WA: The International Society for Optical Engineering, 2004., p.1370, W. A. Traub, ed., pp. 1370-+, Oct. 2004.

8. J. Sturmann, T. A. ten Brummelaar, S. T. Ridgway, M. A. Shure, N. Safizadeh, L. Sturmann, N. H. Turner, and H. A. McAlister, "Infrared beam combination at the CHARA array," in Interferometry for Optical Astronomy II. Edited by Wesley A. Traub . Proceedings of the SPIE, Volume 4838, pp. 1208-1215 (2003)., W. A. Traub, ed., pp. 1208-1215, Feb. 2003.

9. D. H. Berger, D. R. Gies, H. A. McAlister, T. A. ten Brummelaar, T. J. Henry, J. Sturmann, L. Sturmann, N. H. Turner, S. T. Ridgway, J. P. Aufdenberg, and A. M. Mérand, "First Results from the CHARA Array. IV. The Interferometric Radii of Low-Mass Stars," ApJ , 2006. In press.

10. D. H. Berger, "Longitudinal dispersion compensation for a long baseline optical interferometer," Ph.D. Thesis , Dec. 2003.

11. A. C. Oliveira, L. S. de Oliveira, and J. B. dos Santos, "Studying focal ratio degradation of optical fibres with a core size of $50 \mu \mathrm{m}$ for astronomy," MNRAS 356, pp. 1079-1087, Jan. 2005. 